\title{
Handover in a mobile wireless communication network -A Review Phase
}

\author{
Rebin Abdullah Saeed a, \\ a PG Student, Department of Information Technology, Lebanese French University, Erbil, Kurdistan Region, Iraq
}

*Corresponding Author rebin.abdullah88@gmail.com (Rebin Abdullah Saeed)

Received : 18 04 2019 Accepted : 20 05 2019

\begin{abstract}
Mobility is the features of mobile communication that makes it desirable by all and varied. The whole world is now attractive in wireless communication as it provides users' ability to communicate on -the -go. IT is accomplished by transferring users from a radio network to another. This process is called handover. Handover occurs by adjusting the duration of soft handovers, the size of the areas and either by cell crossing or by deterioration in the signal quality of the current channel - the brief overview of handover, Handover in WiMAX and LTE, types of handover, handover types solutions, usually used handover parameters, some methods employed in the literature and I contemporary the convergent fact for continuance in the area of mobile wireless communication Handover.
\end{abstract}

Keywords: Wireless Communication Network, WiMAX, LTE, Handover, GSM and CDMA

\section{Introduction}

Handover is a progression in mobile communications and telecommunications in which a connected a data session, or cellular call is transferred from one cell site (base station) to another without disconnecting the session. Cellular services are based on handover and mobility, permitting the user to be progressed from one cell position range to another or to be moved to the nearest cell position for better action [1, 2, 3]. Handover is an essential element in deploying and planning cellular networks. It lets users create connect phone or data sessions calls on the move. This process keeps the calls and data sessions connected even if users move from one cell position to another. Mobility is the main features that made wireless cellular communication system an indispensable. Handover is obligatory during signal quality deprivation or/and cell crossing in the current channel. And also, we need handover for user performance and mobility. During handover, there is switching among thereby transfer users and networks to another network or Base Station [4]. The essential of 5G is heterogeneous networks and in such networks, seamless handover in immutable. Phases of handover are, decision, execution and: discovery. Network discovery finds a suitable network that satisfies user desired Quality of Services (QoS). Decision phase is when the handover should take place (also known as a handover initiation phase). These phases regulate seamlessness of the handover. Wrong time of initiation leads to unnecessary handover or call drop rate is increased and thereby result in poor QoS. Therefore, handover should take place at the right time by trigger handover decision considering all the parameters properly to ensure QoS is not affected and unnecessary handover is avoided.

\section{The classification of Handover is based on the} following factors

\subsection{Access Technology}

\subsubsection{Horizontal Handover}

A horizontal handover is a traditional handover. Horizontal handovers are Layer 2 handovers. Here, only the BS is changed, and IP information is maintained. Here, MS uses hard handover (HHO, break before make handover) when moving to another BS. all connections are broken at all layers and no context information is shared between BSs. Latency is on the order of around 1000ms. Recent WiMAX standard also supports soft handover (SHO, make before break) and Fast BS Switching (FBSS) $[5,6,7]$.

\subsubsection{Vertical Handover}

There are also inter -technology handovers where a call's connection is transferred from one access technology to another, e.g., a call is transferred from Group Special Mobile (GSM) to Universal Mobile Telecommunications System (UMTS) or from Code Division Multiple Access (CDMA IS 95) to cdma2000. This is also called inter-technology handover, and it is a handover that occurs between two networks of different technology, e.g., 3G to 4G. Vertical handover requires both layer 2 (Data Link layer) and layer 3 to complete the handover procedure successfully $[8,9]$. 


\section{Protocol Layers Involved}
- Cross Layer Based handover
- Data link Layer $\sim$ Based handover
- Network Layer Based handover

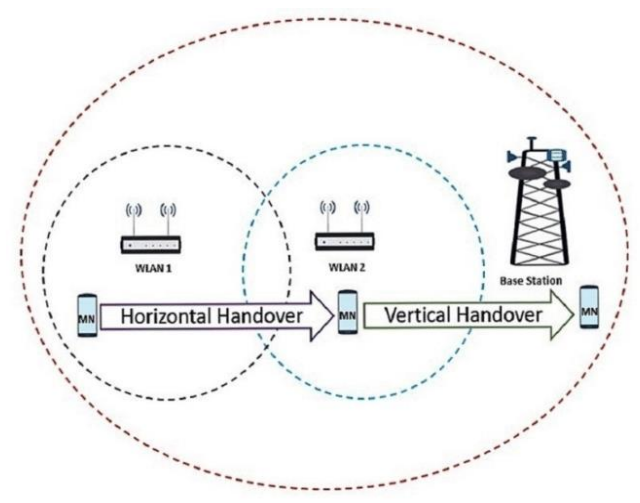

Fig1.Horizontal and Vertical Handover

4. Type of Technology that the Network supports

4.1 Hard handover

\subsubsection{Before $\mathrm{HO}$}

- Network Topology Advertisement

- MS scanning of neighbour BSs
- Association procedure

\subsubsection{During HO}

- Cell Reselection

- HO decision \& initiation

- Synchronize with new downlink and obtain parameters

- Obtain uplink parameters

- Ranging and uplink parameter adjustment

- MS re authorization

- Re register

- Termination with the serving BS

\subsection{Soft Handover}

Soft Handover is similar to HHO but with one significant difference; with SHO an MS is registered to multiple BSs (the Active Set) at the same time. During SHO two or more BSs are sending and receiving the same information to the MS. It utilizes more resources [10, 11].

\subsection{Softer Handover}

This is slightly the same as soft handover except that it occurs between two different sectors of the same cell.

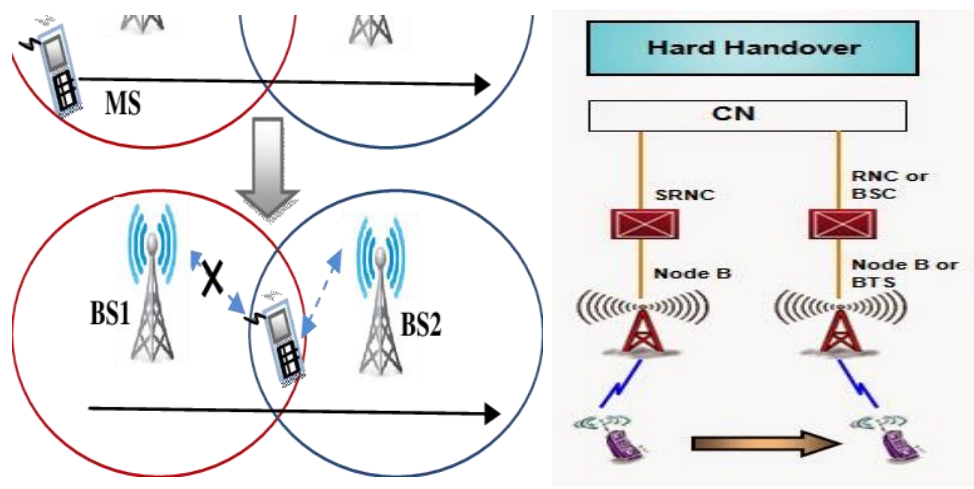

Fig2. Hard Handover Mechanism

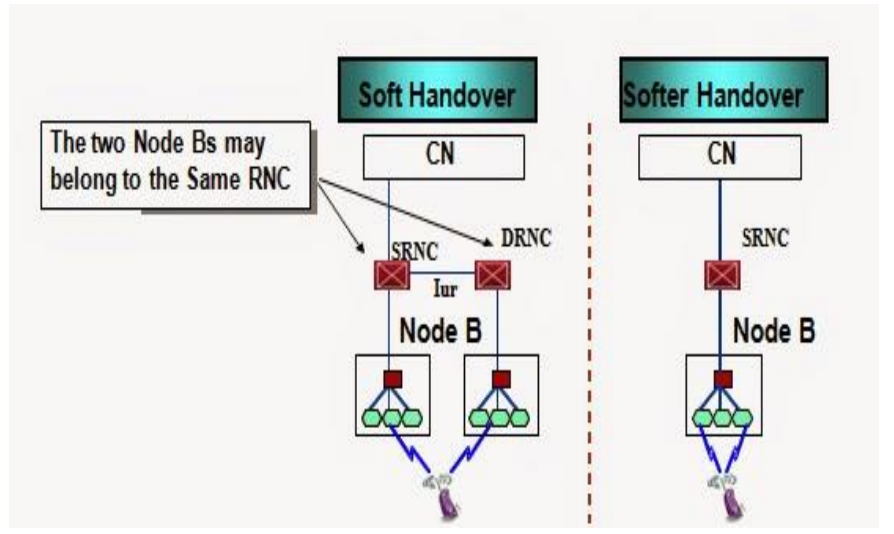

Fig3. Soft and Softer Handover 


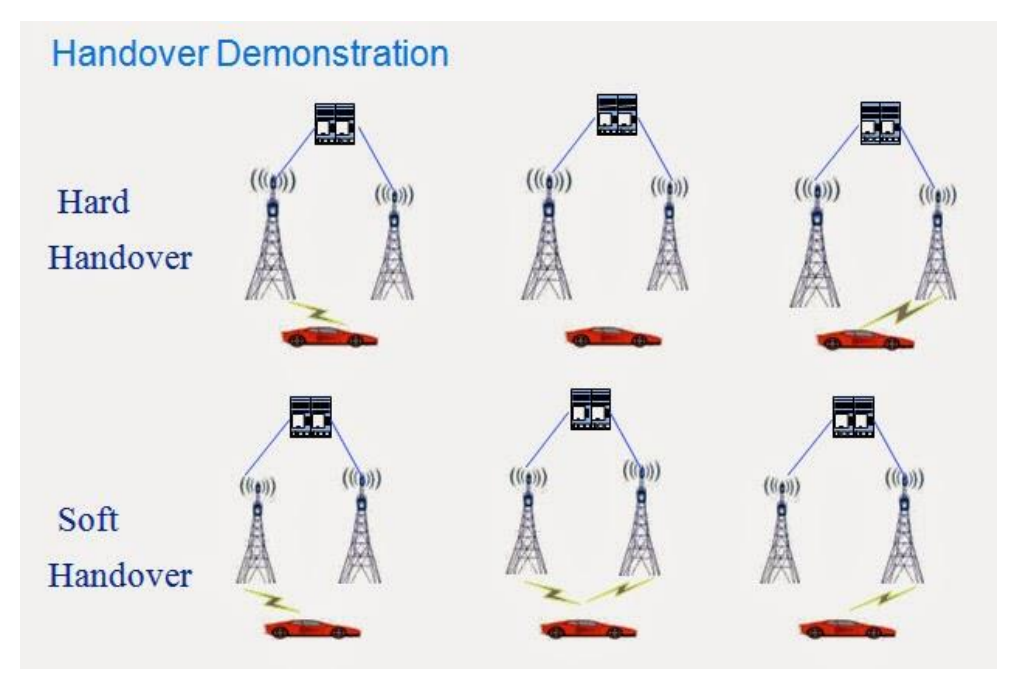

Fig4. Hard Handover

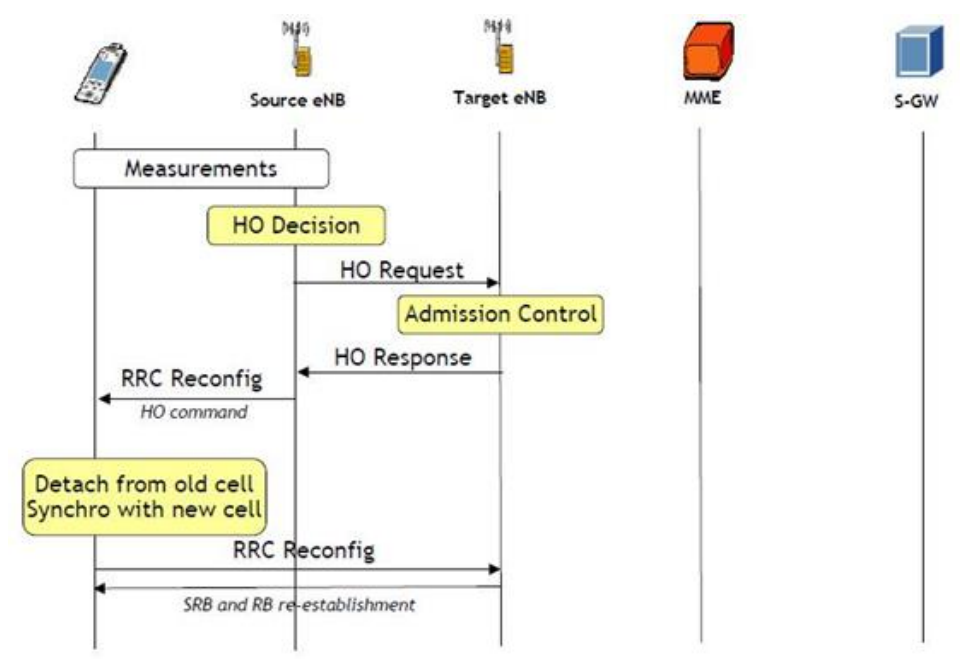

Fig5.Handover Process in LTE

\section{Different Handover Mechanisms in the LTE Network}

In Handover Parameter Optimization (HPO), LTE system Handover performance can be improved by (HPO). Load Balancing (LB) is an instrument that advances the user's fulfilment. LB may result in the rise of the frequency of inter cell handover and henceforth increase the opportunity of handover glitches leading to discount in handover performance. Also, the techniques to improve both HPO and LB don't consider the network permitted maximum radio link failure (RLF) ratio. Radio link failure would raise the possibility of call dropping. The (DHA) dynamic hysteresis adjusting method be able to be used to develop handover performance and the number of fulfilled users in LTE networks. Dynamic Hysteresis Adjusting uses the allowed extreme RLF ratio as a key indicator [12].

One of the main objectives of LTE or any wireless system for that matter is to provide seamless and fast handover from one cell (a source cell) to another (a target cell). The service should be maintained during the handover procedure; data transfer would not be postponed or would not be missing; otherwise, performance will be dramatically tainted.

In the design of IMT Advanced systems, the flexibility to support various $4 \mathrm{G}$ deployments and meeting the latency requirement on handover is significant. In the cellular network mobility management, handover is one of the primary mechanisms. The IMT -Advanced systems are designed based next generation WiMAX networks and 3GPP LTE Advanced with a focus on IEEE $802.16 \mathrm{~m}$. Various handover schemes are being developed, proposed, and analyzed by IEEE $802.16 \mathrm{~m}$ and 3GPP to minimize handover latency and to fulfill the requirement for quality of service during handover [13].

\section{Handovers in Mobile WiMAX}

A great necessity of a mobile device is the capability to change its serving base station if there exists another base station with best signal strength in the reach of a mobile station (MS). Handovers are a process that offers continuous connection when a Mobile Station travels from the air 
interface of one Base Station to another air interface provided by another Base Station (BS) without troubling the existing connections. Handovers are needed to support mobility. For a handover to occur, one needs to have at least two base stations: serving base station (SBS) and target base station (TBS). The handovers have usually measured a change in serving the base station, but it does not inherently mean that the BS must be changed $[14,15]$.

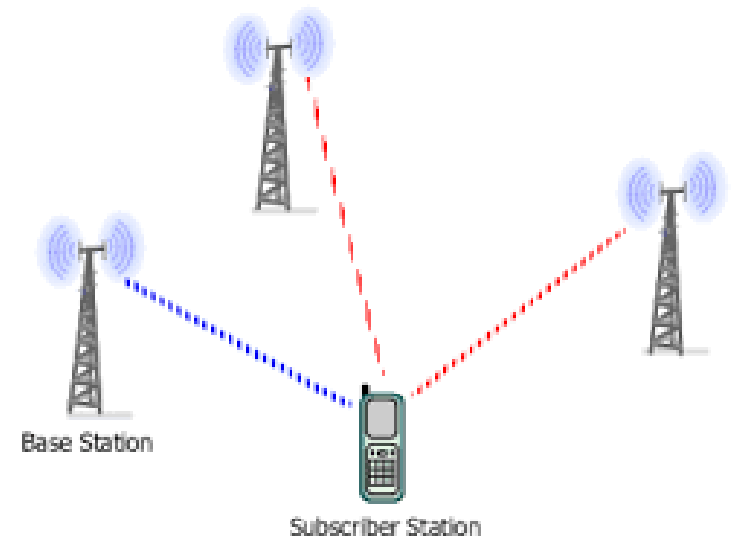

Fig6.Handovers in Mobile WiMAX

\subsection{Latency and Handover}

Handover is machinery to maintain uninterrupted user communication session during a user's movement from one location to another. While the Mobile Station (MS) travels to another cell and performs handover, the service packets for the MS will be delayed, and the service might be disrupted for some time. This delay is known as Latency time.

For non real time services such as e mail or file transfer, Latency time is not an issue. However, the delay sensitive applications, such as video streaming service, should be delivered within the delay of 20 to $25 \mathrm{~ms}$. If the transmission delay of real-time packets is longer than the playout delay, those packets will be discarded, and packet loss probability is increased.

\subsection{Handover Procedure}

For a handover process, the Serving BS broadcasts information message, typically using MOB NBR ADV, that contains information of neighbor BSs. The MS scans the neighbor BSs periodically and selects the target BS candidates, by the quality of signals or other parameters and sends a handover request message to the serving BS. The Serving BS then exchanges the handover messages with the target BSs candidates and finally selects a target BS. It sends the handover response message to the MS. The MS, on the reception of the message, breaks the connection with the serving BS and makes the connection with target BS and performs the network re entry process. The Target BS gets the security information of the MS from the serving BS. MS can receive or send traffic after the network re entry process. The network re entry process consists of synchronization with the new downlink, ranging and synchronization with uplink, reauthorization and re registration procedures. After breaking the connection with Serving BS, the MS synchronizes with the new downlink of Target BS to obtain DL and UL transmission parameters. The MS conducts ranging process to acquire correct timing offset and power adjustments. For this ranging process, Target BS uses Fast Ranging IE to provide a non contention based ranging opportunity to the MS. If the MS does not receive the Fast Ranging IE message, it conducts a contention based ranging process by transmitting the CDMA codes.

\subsection{Handover Mechanisms}

The HO latency remains an issue that affects real time applications. Many researchers have introduced proposals to improve the $\mathrm{HO}$ mechanism for real-time applications. This section reviews some of the proposals for improving the $\mathrm{HO}$ mechanism.

\section{Fast Handover Algorithm for IEEE 802.16e Broadband Wireless Access System}

Lee presented fast $\mathrm{HO}$ algorithm for IEEE 802.16e BWA to reduce the waste of the wireless channel resources and the HO latency. His proposed algorithm includes Target BS Selection (TBS), Fast Synchronization and Association (FSA) and Optimized HO Initiation Timing (OHIT). Here HO operation time is analyzed with four different HO scenarios. It will result to increase system throughput and certify the efficiency of the proposed algorithm. Some parameters of the scheme depend on the backbone network traffic condition, cell loading rate, user mobility, and CINR.

- Type 1 is contention based ranging with pre association.

- Type 2 is contention based ranging without pre association.

- Type 3 is non contention based ranging with pre association.

- Type 4 is non contention based ranging without pre association.

\section{Fast Base Station Switching in Soft Handover}

We are considering a fast base station switching technique. In this method, a diversity set is maintained for each mobile station. Diversity set is maintained by both the mobile station and serving base station. In fig. 7 , the mobile station selects one base station from diversity set is a collection of base stations that can be chosen as target base station for a handover. Diversity set as presenter base station sends its current location to it which is sent to base station controller for the decision of a handover. The serving base station and mobile station monitors the neighboring base stations that can be added in diversity set. Whenever there is a need of handover base station controller sends handover 
initiation message to the mobile station. Handover decision can be taken by the mobile station, base station or base station controller depending upon the implementation.

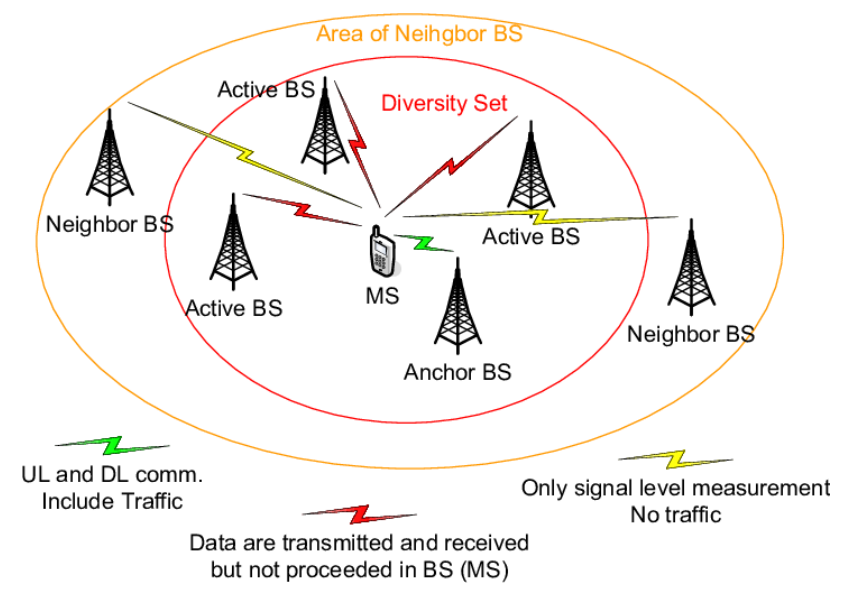

Fig7. Fast Base Station Switching

\section{General Procedure of Handover Control}

- Measuring

- The measurement objects are decided by Remote Network Support (RNC). Usually, either Ec/Io or RSCP (Received Signal Code Power) of the P $\sim \mathrm{CPICH}$ channel is used for handover decision.

- $\quad \mathrm{RNC}$ adopts Ec/Io (Energy per chip to Interference power ratio) measurement because Ec/Io embodies both the received signal strength and the interference. The relation of $\mathrm{Ec} / \mathrm{Io}$ and $\mathrm{RSCP}$ is shown as follows:

- $\mathrm{Ec}_{\mathrm{C}} / \mathrm{Io}=\mathrm{RSCP} / \mathrm{RSSI}$

- In the above equation, RSSI (Received Signal Strength Indicator) is measured within the bandwidth of associated channels

- Filtering

- The measurement results should be filtered before being reported. Measurement filtering can be regarded as a low pass filtering procedure. The following equation is applied for filtering.

- $\mathrm{Fn}=(1 \sim \mathrm{a}) \mathrm{Fn} \sim 1+\mathrm{a}^{*} \mathrm{Mn}$

- Variants definition :

- Fn: filtered measurement result ;

- Fn 1: last filtered measurement result ;

- Mn: latest Ec/Io or RSCP measurement result received from the physical layer;

- $\mathrm{A}=1 / 2(\mathrm{k} / 2), \mathrm{k}$ means the "Filter coefficient," which is included in the Measurement Control message. It is decided by the UTRAN.

- FO is initialized by the first measurement result M1.
- Reporting

- The period report triggered handover

- Base on the filtered measurement result

- Event report triggered handover

- $\quad$ Base on the event

\begin{tabular}{|l|l|}
$\left.\begin{array}{l}\text { Soft } \\
\text { Handover }\end{array}\right\}$ & $\begin{array}{l}\text { Period } \\
\text { Handover }\end{array}\left\{\begin{array}{l}\text { Measurement result filtered in UE } \\
\text { Event decided in RNC } \\
\text { Handoverdecided in RNC }\end{array}\right.$ \\
Event & $\left\{\begin{array}{l}\text { Measurement result filtered in UE } \\
\begin{array}{l}\text { Event decided in UE } \\
\text { Handoverdecided in RNC }\end{array}\end{array}\right.$ \\
\hline
\end{tabular}

- Handover Algorithms

- All the handover algorithms containing soft handover, hard handover, and others are applied to the event decision made according to the quantity reports.

- Events defined in 3GPP specifications

- Intra-frequency events : $1 \mathrm{~A} \sim 1 \mathrm{~F}$

- Inter-frequency events : $2 \mathrm{~A} \sim 2 \mathrm{~F}$

- Inter $\mathrm{RAT}$ events : 3A 3D

- Note: RAT is short for "Radio Access Technology," e.g., UMTS\&GSM

\section{Concepts Related to Handover}

- Active Set:

- A set of cells that have established radio links with a specific mobile station.

- User information is sent from all these cells.

\begin{tabular}{c|l}
\hline Event & \multicolumn{1}{c}{ Description } \\
\hline 1A & $\begin{array}{l}\text { Quality of target cell improves, entering a } \\
\text { report range of relatively activating set quality }\end{array}$ \\
\hline 1B & $\begin{array}{l}\text { Quality of target cell decreases, depart from a } \\
\text { report range of relatively activating set quality }\end{array}$ \\
\hline 1C & $\begin{array}{l}\text { The quality of a non-activated set cell is better } \\
\text { than that of a certain activated set cell }\end{array}$ \\
\hline 1D & Best cell generates change \\
\hline 1E & $\begin{array}{l}\text { Quality of target cell improves, better than an } \\
\text { absolute threshold }\end{array}$ \\
\hline 1F & $\begin{array}{l}\text { Quality of target cell decreases, worse than } \\
\text { an absolute threshold }\end{array}$
\end{tabular}

Fig8. Soft Handover Event

- Monitored Set:

- A set of cells that are not in the active set but are monitored according to the list of adjacent cells assigned by the UTRAN. 
- $\quad$ Detected Set:

- A set of cells that are neither in the active set nor in the monitor set.

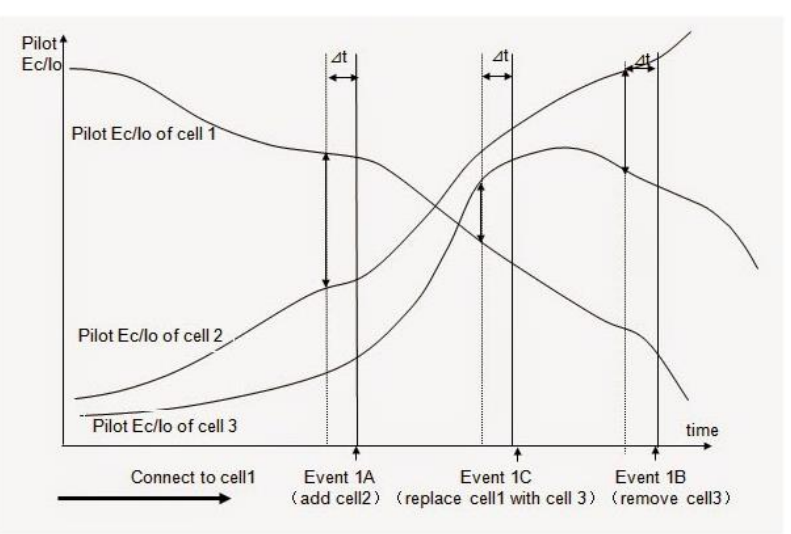

Fig9. An Example of SHO Procedure

\section{Simulation model}

Consider the handover scenario of Fig. 11 in which the MN is moving away with the velocity 'v' from the Global System of Mobile Communication (GSM) network domain to the WiMAX network domain. The distance between two BSs is $\mathrm{D}=\mathrm{R} 1+\mathrm{R} 2 \mathrm{~L}$ where $\mathrm{R} 1$ and $\mathrm{R} 2$ are radii of $\mathrm{BS} 1$ and $\mathrm{BS} 2$ respectively. $\mathrm{L}$ is the overlapping distance of two BSs. The received signal strength by $\mathrm{MN}$ from $\mathrm{BSi}$ is

$$
\mathrm{Si}(\mathrm{k})=\mathrm{Pt}+\mathrm{Gt}-\mathrm{Lt}-\mathrm{PLi}(\mathrm{k})+\mathrm{Gr}-\mathrm{Lr}
$$

Where Pt is transmitted signal strength from a BS. Gt, Lt and Gr, Lr are antenna gains and losses of transmitter and receiver respectively. PLi(k) represents path loss component at Kth sampling distance from BSi. The Okumura Hata propagation model is considered for the GSM network. The path loss equation is given by

Pli $(\mathrm{k})=69.55+26.16 \log \mathrm{fi}-13.82 \log \mathrm{hb}-\alpha(\mathrm{hm})+$ $(44.9-6.55 \log \mathrm{hb}) \log \mathrm{di}(\mathrm{k})$
For WiMAX network, an extended version of Erceg model is used an equation for calculating path loss in decibels is given by

$$
\operatorname{Pli}(\mathrm{k})=\mathrm{A}+10 \gamma \log 10(\mathrm{di}(\mathrm{k}) / \mathrm{dO})+\mathrm{Zi}(\mathrm{k})+\mathrm{PLf}+\mathrm{PLhms}+\mathrm{PL} \theta \mathrm{ms}
$$

Where $\mathrm{d}_{\mathrm{i}}(\mathrm{k})$ is the distance between $\mathrm{BS}_{\mathrm{i}}$ and $\mathrm{MN}$ at $\mathrm{k}^{\text {th }}$ Sampling instant in meters and $\mathrm{Z}_{\mathrm{i}}(\mathrm{k})$ is shadow fading component at $\mathrm{k}^{\text {th }}$ sampling instant, which is log normally distributed and is described (in $\mathrm{dB}$ ) as a Gaussian distribution with zero mean and standard deviation $\sigma_{i}$.

$$
\mathrm{Z}_{\mathrm{i}}(\mathrm{k})=\rho_{\mathrm{i}} \mathrm{Z}_{\mathrm{i}}(\mathrm{k} \sim 1)+\sigma_{\mathrm{i}} \sqrt{1-p i^{2}} \eta_{\mathrm{i}}(0,1)
$$

Where $\rho \mathrm{i}$ is correlation coefficient of $\{\mathrm{Zi}(\mathrm{k})\}$. ni $(0,1)$ are standard random variables with zero mean and unity variance. Parameter A represents the free space path loss and is given by

$$
\mathrm{A}=20 \log _{10} \frac{4 \pi d 0}{\gamma}
$$

$\gamma$ represents path loss exponent defined as

$$
\begin{aligned}
& \gamma=\mathrm{a}-\mathrm{bhb}+\frac{\mathrm{c}}{\mathrm{hb}} \\
& \mathrm{PL}_{\mathrm{f}}=6 \log \frac{\mathrm{fi}}{1900} \\
& \mathrm{PL}_{\mathrm{hms}}=\sim 20 \log \left(\frac{\mathrm{hm}}{2}\right) \text { for Suburban areas } \\
& \mathrm{PL}_{\mathrm{qms}}=0.64 \ln \left(\frac{\mathrm{a}}{360}\right)+0.54\left(\ln \left(\frac{\mathrm{a}}{360}\right)\right) 2
\end{aligned}
$$

Where $\mathrm{fi}$ is the carrier frequency and bhb is the base station antenna height in meters. The empirical constants used in the above equations are well defined for suburban areas in Erceg model as given in Table 1.PLF, $\mathrm{PL}_{\mathrm{hms}}, \mathrm{PL}_{\mathrm{qms}}$ are correction factors corresponding to frequency $\mathrm{f}_{\mathrm{i}}, \mathrm{MN}$ height ${ }_{\mathrm{m}} \mathrm{HM}$, and $\mathrm{MN}$ antenna directivity $q . \mathrm{PL}_{\mathrm{qms}}$ is often referred to as the antenna gain reduction factor and accounts for the fact that the angular scattering is reduced owing to the directivity of the antenna. $\alpha\left(\mathrm{h}_{\mathrm{m}}\right)$ is the antenna correction factor for an urban area in $\mathrm{dB}$ and is given by
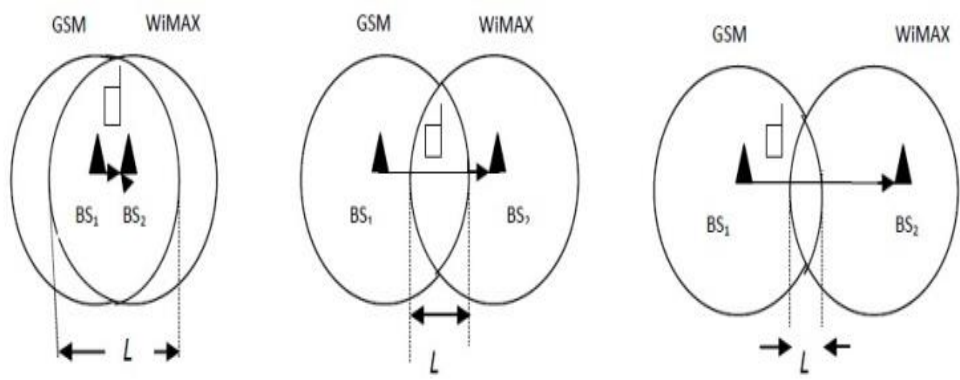

Fig10. Handover Scenario

Table1. Simulation Parameters

\begin{tabular}{|c|c|c|c|}
\hline $\mathrm{R}_{1}=5000 \mathrm{~m}$ & Radius of GSM cell & $\mathrm{L}_{\mathrm{t}}=3 \mathrm{~dB}$ & Transmitter antenna loss \\
\hline $\mathrm{R}_{2}=5000 \mathrm{~m}$ & Radius of WiMAX cell & $\mathrm{G}_{\mathrm{r}}=0 \mathrm{~dB}$ & Receiver antenna gain \\
\hline $\mathrm{d}_{\mathrm{o}}=100 \mathrm{~m}$ & Correlation distance & $\mathrm{G}_{\mathrm{t}}=8 \mathrm{~dB}$ & Transmitter antenna loss \\
\hline
\end{tabular}




\begin{tabular}{|c|c|c|c|}
\hline $\mathrm{P}_{\mathrm{t}}=43 \mathrm{dBm}$ & Transmitter power & $\sigma_{\mathrm{i}}=8 \mathrm{~dB}$ & $\begin{array}{c}\text { Standard deviation of shadow } \\
\text { fading }\end{array}$ \\
\hline $\mathrm{V}=(1 \sim 25) \mathrm{m} / \mathrm{s}$ & Velocity of $\mathrm{MN}$ & $\mathrm{h}_{\mathrm{b}}=50$ meter & Base station antenna height \\
\hline $\mathrm{q}=10$ & $\begin{array}{c}\text { MN antenna } \\
\text { directivity }\end{array}$ & $\mathrm{h}_{\mathrm{m}}=2$ meter & Mobile station antenna height \\
\hline$\rho_{\mathrm{i}}=0.35$ & Correlation coefficient & $\begin{array}{c}\mathrm{f}_{1}=900 \\
\mathrm{MHz}\end{array}$ & Carrier Frequency of GSM \\
\hline $\mathrm{G}_{\mathrm{t}}=18 \mathrm{~dB}$ & Transmitter antenna & $\mathrm{f}_{2}=900$ & Carrier Frequency of WiMAX \\
& gain & MHz & \\
\hline $\mathrm{T}_{\mathrm{s}}=10 \mathrm{~ms}$ & Sampling time & $\mathrm{S}_{\mathrm{th}}=-100$ & Threshold Signal Strength \\
\hline $\mathrm{a}=3.6$ & $\mathrm{~b}(\mathrm{~m})=0.005$ & $\mathrm{c}\left(\mathrm{m}^{-1}\right)=20$ & \\
\hline
\end{tabular}

\section{Conclusion}

I have offered the list of some methods been employed so far in the area of Mobile communication handover, and It is worth mentioning that Quality of Services (QoS) in mobile communication is knotted mostly to successful and how fast the handover is carried out. It is also well known that the soft computing based algorithm performed better due to the aptitude of the soft computing method to grip the uncertainty embedded in the mobile wireless communication network. Consequently, there is a need for more seamless handover by selecting multiple and appropriate network parameters.

\section{Reference}

[1] Kai Niu, Wu. Weiling, Secrecy Cognitive Gain of Cognitive Radio Sensor Networks with Primary Outage Constraint; Mobile Communication principle, Beijing, Publishing house of the electronics industry, 116 (2005)

[2] Jiang Xie, Weiyi Zhao. Inter gateway Cross layer Handoffs in Wireless Mesh Networks, IEEE Proc. Globecom, 4 (2009) 1 6.

[3] S. Brahmjit, Outage probability analysis in soft handover for $3 g$ wireless networks, $3 G$ and Beyond, $6^{\text {th }}$ IEEE Inte. Conf. (2005) 1 5

[4] S. Migaldi, L. Eastwood, V. Gupta, Qiaobing Xie., Mobility using IEEE 802.21 in a heterogeneous IEEE 802.16/802.11 based, IMT advanced (4G) network, IEEE Wireless Comm. , (2008) 26 34.

[5] V. Mhatre, K. Papagiannaki, Using smart triggers for improved user performance in 802.11 Wireless Networks, Proc. $4^{\text {th }}$ inter. conf. Mobile systems, appl. serv, ACM Mobisys'06, (2006) 246 259.
[6] N. Golmie, S. Woon, Y. A Sekercioglu, Effective link triggers to improve handover performance, IEEE PIMRC 06, (2006) 1 5.

[7] S. Brahmjit, K. Shakti, K. Aggarwal, Handover Initiation Control Techniques in Mobile Cellular Systems, IETE Tech. Rev., 20 (2003) 13 21.

[8] L.Y. Azita, Y. Norsuzila, T. A. Mohd, (2011): Handover Initiation Across Heterogeneous Access Networks for Next Generation Cellular Network, 2011 IEEE Symposium on Wireless Technology and Applications, 9 12 .

[9] D. Cypher, S. Yoo, N. Golmie, Predictive Link Trigger Mechanism for Seamless Handovers in Heterogeneous Wireless Networks, Wirel Commun Mob Com., 9 (2009) 685 703.

[10]J. Shucong, L. Wenyu, D. Xiaoyu, Z. Lin, L. Yu, L. Jiaru, A Dynamic Hysteresis adjusting Algorithm in LTE Self Organization Networks, IEEE $75^{\text {th }}$ Vehicular Tech. Conf. (2012).

[11]Y. K. Ronny, J. Inuk, Y. Xiangying, Ch.Chao Ch, Advanced Handover Schemes in IMT Advanced Systems, IEEE Communications Magazine, (2010).78 85.

[12] Kwan Cheng Chen, J. Roberto De Marca, Mobile WiMAX, Wiley IEEE Press (2008)5 6.

[13]S. Choi, T. Kwon and G.H. Hwang, Fast handover scheme for real time downlink services in IEEE 802.16e BWA system. In IEEE VTC, 3(2005) 2028 2032.

[14] K. Kyamakya, Lee, D.H., and J.P. Umondi, Fast Handover Algorithm for IEEE 802.16e Broadband Wireless System, IEEE Xplore, (2006). 
[15]Zdenek BECVAR, Pavel MACH, Robert BESTAK, Initialization of Handover Procedure in WiMAX Networks, ICT Mobile Summit 2009 Conference Proceedings, Inter. Infor. Manage. Corp. (2009).

\section{Author Biography}

Robin A. Saeed. Was born in 1988 in Erbil, Kurdistan Region, Iraq. He completed his bachelor degree in information technology from Tishk International University in 2013. Currently he is pursuing master degree at Lebanese French University. His area of interest image processing and wireless networks.

\section{About The License}

(C) 2019 The Authors. This work is licensed under a Creative Commons Attribution 4.0 International License which permits unrestricted use, provided the original author and source are credited. 